\title{
COINCIDENT PAIRS OF CONTINUOUS SECTIONS IN PROFINITE GROUPS
}

\author{
H. APPELGATE AND H. ONISHI
}

\begin{abstract}
Given a profinite group $G$ and a closed subgroup $H$, we show there exist continuous sections $s:(G / H)_{1} \rightarrow G$ and $s^{\prime}:(G / H)_{r} \rightarrow G$ of the projections $G \rightarrow(G / H)_{1}$ and $G \rightarrow(G / H)_{r}$ onto the left and right coset spaces, respectively, $\operatorname{such}$ that $\operatorname{im}(s)=\operatorname{im}\left(s^{\prime}\right)$.
\end{abstract}

1. Coincident pairs of sections. Given a group $G$ and a subgroup $H$, let $(G / H)_{1}$ and $(G / H)_{r}$ denote, respectively, the left and right coset spaces of $H$ in $G$. A pair of sections

$$
(G / H)_{1} \stackrel{s}{\rightarrow} G \stackrel{s^{\prime}}{\leftarrow}(G / H)_{r}
$$

will be called coincident if $\operatorname{im}(s)=\operatorname{im}\left(s^{\prime}\right)$. Having a coincident pair of sections is equivalent to having a subset $S$ of $G$ such that

$$
G=\coprod_{x \in S} x H=\coprod_{x \in S} H x \quad \text { (disjoint union) }
$$

( $\left.S=\operatorname{im}(s)=\operatorname{im}\left(s^{\prime}\right)\right)$ or to having a 1-1 correspondence $a H \leftrightarrow H b$ between $(G / H)_{1}$ and $(G / H)_{r}$ such that $a H \cap H_{b} \neq \varnothing$.

The following criterion is easy to establish (see [1]).

LEMMA 1. There exists a coincident pair of sections for $G \bmod H$ if and only if for every $a$ in $G,\left(H: H \cap a H a^{-1}\right)=\left(H: H \cap a^{-1} H a\right)$ where, in general, $(G: H)$ denotes the cardinality of $G / H$ (left or right coset space).

Not every $G \supset H$ will admit a coincident pair of sections. We thank our colleague Peter Stebe for the following example. Let $G=\langle a, b\rangle$ be the free group on two generators $a, b$ and $H$ the subgroup generated by $a^{n} b a^{-n}$ for all $n \geqslant 0$. Then $a H a^{-1}<H$ (proper subgroup), $H<a^{-1} H a$ and ( $H: H$ $\left.\cap a^{-1} H a\right)>1$ but $\left(H: H \cap a H a^{-1}\right)=1$.

When $(G: H)<\infty$ there is always a coincident pair of sections. More generally, if $N(H)$ is the normalizer of $H$ and $G$ is torsion $\bmod N(H)$, i.e. for every $x \in G, x^{n} \in N(H)$ for some $n>0$, then there exists a coincident pair of sections. This can be seen using the following criterion. Given an inner automorphism $f: G \rightarrow G$ let $H_{0}=H, H_{n}=f^{-n}(H) \cap H_{n-1}$ for $n>0$. If for

Received by the editors December 30, 1974.

AMS (MOS) subject classifications (1970). Primary $20 \mathrm{~F} 20$. 
every $f$ there exists $n>0$ such that $\left(H_{n-1}: H_{n}\right)=\left(H_{n-1}: f\left(H_{n}\right)\right)$, then there exists a coincident pair of sections for $G \bmod H$. This criterion follows from Lemma 1 and the observation that for any subgroups $K, L$ of $H,(K: K \cap L)$ $=(L: K \cap L)$ implies $(H: K)=(H: L)$.

For finite groups $G$, one can prove the existence of coincident pairs of sections by applying the following well-known result from combinatorics. For a proof see, e.g., [2, pp. 50-51].

LeMma 2. If $A$ is a nonempty finite set and $A=\amalg_{i \in I} B_{i}=\amalg_{i \in I} C_{i}$ are two partitions of $A$ with $\left|B_{i}\right|=\left|C_{i}\right|=r(|B|$ denotes number of elements) for all $i$ in the finite index set $I$, then there is a bijection $i \mapsto j$ of $I$ onto itself such that $B_{i} \cap C_{j} \neq \varnothing$.

We will make use of this result later on.

2. Coincident pairs of sections in profinite groups. Let $G$ be a profinite group and let $U$ range over the open normal subgroups of $G$ (for definitions and basic properties see, e.g., [3, Chapter I]). Then for any closed subgroups $H, K$ of $G, H=\lim _{U} H U / U$ and $H \cap K=\lim _{U}(H U \cap K U) / U$ (lim denotes projective limit). Using these facts, it is not hard to prove $\left(H: H \cap a H a^{-1}\right)$ $=\left(H: H \cap a^{-1} H a\right)$ for any $a$ in $G$. Thus, by Lemma 1 , there exists a coincident pair of sections for $G \bmod H$.

On the other hand, it is well known that for any closed subgroup $H$ of $G$ there exists a continuous section $G / H \rightarrow G$. Our objective is to prove the following

THEOREM. If $G$ is a profinite group, then for any closed subgroup $H$ of $G$ there exists a coincident pair of continuous sections for $G \bmod H$.

Proof. Let $\delta$ be the set of all triples $\left(N, s, s^{\prime}\right)$ where $N$ is a closed normal subgroup of $G$ and

$$
(G / H N)_{1} \stackrel{s}{\rightarrow} G / N \stackrel{s^{\prime}}{\leftarrow}(G / H N)_{r}
$$

is a coincident pair of continuous sections. By taking $N=G$ we see that $\delta \neq \varnothing$.

Given $\left(N, s, s^{\prime}\right)$ and $\left(K, t, t^{\prime}\right)$ in $\delta$, let $\left(N, s, s^{\prime}\right) \leqslant\left(K, t, t^{\prime}\right)$ if $N \supset K$ and the two squares

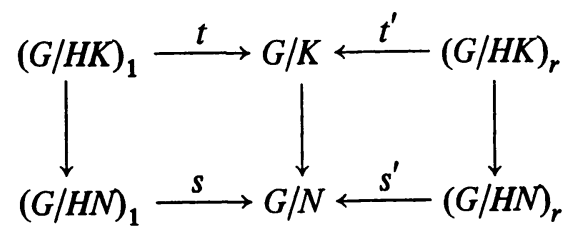

are commutative; the vertical arrows are projections. Clearly $\leqslant$ is a partial order on $\delta$. Let $\left(N_{i}, s_{i}, s_{i}^{\prime}\right)$ be a chain in $\delta$. Put $N=\bigcap_{i} N_{i}$. Then $H N=\bigcap_{i} H N_{i}$ 
and $G / N=\lim _{i} G / N_{i}$. From these facts we easily get a pair $\left(s, s^{\prime}\right)$ such that $\left(N, s, s^{\prime}\right) \in \delta$ and $\left(N_{i}, s_{i}, s_{i}^{\prime}\right) \leqslant\left(N, s, s^{\prime}\right)$ for all $i$. We can now apply Zorn's lemma.

Let $\left(N, s, s^{\prime}\right)$ be a maximal element of $\delta$. We want to show $N=1$. Suppose $N \neq 1$. Then there exists an open normal subgroup $V$ of $G$ such that $N \nsubseteq V$. Let $N_{1}=N \cap V$. Then $N_{1}<N$ and $\left(N: N_{1}\right)<\infty$. We will produce a pair $\left(t, t^{\prime}\right)$ such that $\left(N_{1}, t, t^{\prime}\right) \in \mathcal{S}$ and $\left(N, s, s^{\prime}\right)<\left(N_{1}, t, t^{\prime}\right)$. Replacing $G / N_{1}$ by $G$, we may assume $N_{1}=1$. Then $N$ is finite. We have the following diagram:

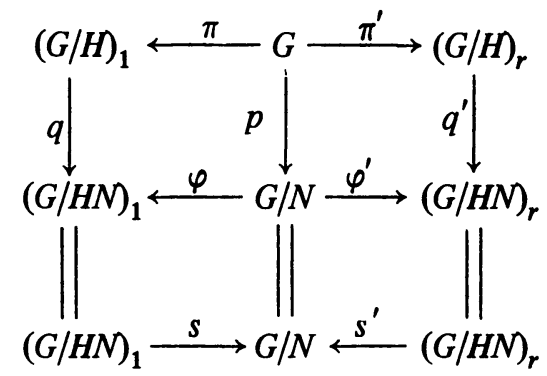

where $\operatorname{im}(s)=\operatorname{im}\left(s^{\prime}\right)=S$, say, and $p, q, q^{\prime}, \pi, \pi^{\prime}, \varphi, \varphi^{\prime}$ are projections, and $s$, $s^{\prime}$ are continuous sections of $\varphi, \varphi^{\prime}$, respectively.

Choose an open normal subgroup $U$ of $G$ such that $N \cap U=1$ and $H N \cap U \subset H$. With some abuse of notation we put $a U N=p(a U), a U H$ $=\pi(a U), H U a=\pi^{\prime}(U a), a U H N=q \pi(a U)$ and $N H U a=q^{\prime} \pi^{\prime}(U a)$ for $a$ $\in G$. Since $N \cap U=1, p$ restricted to $a U$ is a homeomorphism of $a U$ onto $a U N$. Also, since $H N \cap U \subset H, q$ is $1-1$ on $a U H$ and $q^{\prime}$ is 1-1 on $H U a$. Let $K=H \cap N$. Since $N$ is finite, there are $n_{i} \in N, i \in I$ finite, such that $N=\amalg_{i} n_{i} K=\amalg_{i} K n_{i}$. Then for each $A=a N \in G / N, A=\amalg_{i} a n_{i} K$ $=\amalg_{i} K n_{i} a$. Since $A$ is finite and $\left|a n_{i} K\right|=\left|K n_{i} a\right|=|K|$, by Lemma 2, there is a bijection $i \mapsto j$ of $I$ onto $I$ such that $a n_{i} K \cap K n_{j} a \neq \varnothing$.

Now choose $A_{1}, \ldots, A_{m} \in S=\operatorname{im}(s)=\operatorname{im}\left(s^{\prime}\right)$ such that $S \subset \amalg_{k} A_{k} \bar{U}$, where $\bar{U}=U N / N$. Then choose $a_{k} \in G$ such that $A_{k}=a_{k} N$ and choose a bijection $i \mapsto j$ of $I$ onto $I$ depending on $k$ such that $a_{k} n_{i} K \cap K n_{j} a_{k} \neq \varnothing$ and finally choose an element $b_{k i j}$ in $a_{k} n_{i} K \cap K n_{j} a_{k}$. Since $s$ and $s^{\prime}$ are continuous, $s^{-1}\left(A_{k} \bar{U}\right)$ and $s^{\prime-1}\left(A_{k} \bar{U}\right)$ are open and clearly

$$
\begin{array}{ll}
s^{-1}\left(A_{k} \bar{U}\right) \subset a_{k} U H N, & (G / H N)_{Y}=\coprod_{k} s^{-1}\left(A_{k} \bar{U}\right), \\
s^{\prime-1}\left(A_{k} \bar{U}\right) \subset N H U a_{k}, & (G / H N)_{r}=\coprod_{k} s^{\prime-1}\left(A_{k} \bar{U}\right) .
\end{array}
$$

Now let

$$
\begin{gathered}
W_{k i}=a_{k} n_{i} U H \cap q^{-1} s^{-1}\left(A_{k} \bar{U}\right), \\
W_{k j}^{\prime}=H U n_{j} a_{k} \cap q^{\prime-1} s^{\prime-1}\left(A_{k} \bar{U}\right) .
\end{gathered}
$$


Then these are open sets and

$$
(G / H)_{1}=\coprod_{k, i} W_{k i}, \quad(G / H)_{r}=\coprod_{k, j} W_{k j}^{\prime} .
$$

Now let $p_{k i j}^{-1}: A_{k} \bar{U}=a_{k} U N \rightarrow b_{k i j} U$ be the inverse of $p$ on $b_{k i j} U$ and define functions

$$
(G / H)_{1} \stackrel{t}{\rightarrow} G \stackrel{t^{\prime}}{\leftarrow}(G / H)_{r}
$$

by $t=p_{k i j}^{-1} s q$ on $W_{k i}$ and $t^{\prime}=p_{k i j}^{-1} s^{\prime} q^{\prime}$ on $W_{k j}^{\prime}$. Clearly $t$ and $t^{\prime}$ are continuous, $p t=s q, p t^{\prime}=s^{\prime} q^{\prime}$. On each $W_{k i}, q \pi t=\varphi p p_{k i j}^{-1} s q=\varphi s q=q$. Since $\pi t\left(W_{k i}\right)$ $\subset a_{k} n_{i} U H$ and $q$ is $1-1$ on $a_{k} n_{i} U H$, we conclude that $\pi t=\mathrm{id}$, i.e. that $t$ is a section. Similarly $t^{\prime}$ is a section.

Finally, we claim that $\operatorname{im}(t)=\operatorname{im}\left(t^{\prime}\right)$, in fact $t\left(W_{k i}\right)=t^{\prime}\left(W_{k j}^{\prime}\right)$ when $i \mapsto j$ for the given $k$. Let $a=t(a H), a H \in W_{k i}$. Then $a \in b U$, where $b=b_{k i j}$, say $a=b u, u \in U$. Write $b=a_{k} n_{i} x=x^{\prime} n_{j} a_{k}, x, x^{\prime} \in K$. Since $N \cap U=1$, $n u=u n$ for $n \in N$. Thus $a=b u=a_{k} u n_{i} x=x^{\prime} n_{j} a_{k} u$. Thus $s q(a H)$ $=p t(a H)=p(a)=a_{k} u N$ and hence $a=t(a H)=p_{k i j}^{-1} s q(a H)$ $=p_{k i j}^{-1}\left(a_{k} u N\right)$. But since $a_{k} u N \in \operatorname{im}(s)=\operatorname{im}\left(s^{\prime}\right), a_{k} u N=s^{\prime}\left(N H a_{k} u\right)$ $=s^{\prime} q^{\prime}(H a) \in A_{k} \bar{U}$. Since $H a \in H U n_{j} a$, this means that $H a \in W_{k j}^{\prime}$. Therefore $t^{\prime}(H a)=p_{k i j}^{-1} s^{\prime} q^{\prime}(H a)=p_{k i j}^{-1}\left(a_{k} u N\right)=a$. This completes the proof of the theorem.

COROLlaRY. There exists a homeomorphism between $(G / H)_{1}$ and $(G / H)_{r}$ such that the corresponding cosets intersect.

\section{REFERENCES}

1. O. Ore, On coset representatives in groups, Proc. Amer. Math. Soc. 9 (1958), 665-670. MR 20 \#7068.

2. H. Ryser, Combinatorial mathematics, Carus Math. Monographs, no. 14, Wiley, New York, 1963. MR 27 \#51.

3. J.-P. Serre, Cohomologie galoisienne, Lecture Notes in Math., vol. 5, Springer-Verlag, Berlin and New York, 1964. MR 31 \# 4785.

Department of Mathematics, City University of New York, City College, New York, NeW YORK 10031 